\title{
An On-Line Ischemia Monitoring Algorithm for Mobile Devices
}

\author{
L Dranca, A Goñi, J Rodríguez, A Burgos, A Illarramendi \\ University of the Basque Country, Donostia - San Sebastián, Spain
}

\begin{abstract}
The early diagnosis of ischemic events may prevent irreversible damage to the heart muscle. Mobile devices like PDAs provided with wireless communication links are being used successfully in order to build on line monitoring systems. The aim of this paper is to show an ischemia classifier with high rates of sensitivity and positive predictivity and also able to run in real time that can be implemented on a mobile device like a PDA. The LTST database (43 recordings version) has been used to develop and validate the results of the classifier, considering the ischemic and heart rate related events together. The sensitivity of the classifier is $80.65 \%$ for episodes annotated according to $C$ protocol and the positive predictivity is $73.11 \%$ for episodes annotated according to A protocol in the LTST database.
\end{abstract}

\section{Introduction}

Myocardial ischemia is one of the diseases with highest incidence rate in the industrialised countries. Physiologically, is identified by an insufficient oxygenated blood supply compared to current myocardial demand. This event is reflected in a ECG signal as anomalous variations during the ventricular re-polarization. Although the ECG signal analysis is not the most accurate method that exists to detect the ischemic events, it is without question, the least invasive and costly one, and still maintain a high sensitivity level in the detection.

Moreover, prolonged, severe or repeated ischemic episodes can lead to irreversible damages to the cardiac tissue. Therefore it comes up the importance of the early detection of such kind of episodes.

The latest technological advances in the communication and mobile devices have led to significant progresses of the mobile computing area and give the possibility of new any time and anywhere telemonitoring solution. The combination of real time ischemia detection methods with mobile computing techniques may give the solution to the early detection of ischemic events through innovative telemonitoring systems.

The aim of this paper is to present the structure and the validation results of a detection algorithm of transient ischemic events based on ECG signal analysis. The algorithm can be embedded in a mobile such as a PDA and can be executed in real time. Those requirements involve some rules that we had in mind during the development of the algorithm:

1. to be simple enough and with a low computation cost to fulfill the time restrictions of a real time execution in a device with limited computation capabilities and memory capacity (PDA);

2. to generate a minimal number of false alarms

3. to be highly sensitive to ischemic episodes and above all to the most dangerous ones.

We start this paper by mentioning some related work, followed by a description of the transient ischemia detection algorithm. We finish presenting the performances of the algorithm and conclusions.

\section{Related work and materials}

The ischemic transient events are associated with variations (elevations or depressions) of the ST segment in the ECG signal. Nevertheless, similar variations may be also produced by diurnal changes, postural changes, changes in ventricular conduction which make difficult the distinction between ischemic and non-ischemic events. There are several distinct algorithms that address the automatically ischemic detection in the ECG signals applying different methods that consider time domain analysis, KL transform, neural network or fuzzy logic. Nevertheless only a few of them deal explicitly with non-ischemic events such as axis shift events (an interesting reference of the existing ischemia algorithms can be found in [1]). Moreover the majority of the existing algorithms are difficult to be adjusted for real time execution and up to our knowledge, only a few algorithms specially designed to detect ischemic events in real time can be found in the literature [2].

In this paper we propose a new algorithm that detects ischemic events in real time, by using only informations extracted from the ECG recording up to each current analysis point.

In order to encourage the investigation of ischemic/ST events, some ECG databases that contain relevant ST 
changes events have been made available. Among these databases it stands out the Long-Term ST database (LTST) [3] that has been made relatively recently available and that contains ECG recordings from 80 patients covering a larger number of real, day-to-day situations and the corresponding ST segment annotations made by experts. We used the free version of this database to develop and validate our algorithm (43 recordings version).

\section{Algorithm}

The detection algorithm is based on a time domain analysis starting from the ECG samples picked up regularly, from each channel monitored. As the initial annotation protocol followed by the developers of the LTST database was applied independently for each channel, our algorithm follows the same criterion. That means that the analysis is made in parallel, but independently for each channel involved.

The real time monitoring algorithm includes three steps:

1) ECG samples acquisition, 2) a preprocessing phase and 3) a rule-based ischemia detector.

\subsection{ECG samples acquisition}

The ECG samples acquisition part provides the entry data to the rest of the algorithm for its further analysis. In our case it reads the ECG samples from the LTST database recordings with a frequency of 250 samples/second. Therefore it simulates the behaviour of a real ECG sensor.

\section{2. $\quad$ Preprocessing}

The next step, the preprocessing, is the part of the algorithm that works directly with the ECG samples received and provides the parameter values that the ischemia detector uses further. It consists on the application of signal processing methods to the ECG signals in order to identify the beats in the ECG signals and the boundaries of the waves that compose them and to extract the parameter values that are relevant for the rest of the algorithm. For this task we have used the ECGPUWAVE tool [4], a threshold based ECG wave boundaries detector that we previously used successfully in real time ECG analysis applications [5]. For each beat the fiducial point of the QRS, the iso-electric point (considered as the beginning of the QRS complex) and the J-point (considered as the end of QRS complex) are identified. All this values are provided by the ECPUWAVE tool.

Once these points are identified, for each detected beat, the amplitude of the J-point and the amplitude of a point in the ST segment, representing the ST level, are measured. With respect to the election of the ST level measurement point in the ST segment, we follow the protocol specified by the authors of the LTST database [3], that is, the amplitudes were measured at $\mathrm{J}+80 \mathrm{~ms}$ point if the heart rate (HR) was less than 100 beats / minute, $\mathrm{J}+72 \mathrm{~ms}$ if $100 \leq$ $\mathrm{HR}<110, \mathrm{~J}+64 \mathrm{~ms}$ if $110 \leq \mathrm{HR}>120$ and $\mathrm{J}+60 \mathrm{~ms}$ if $\mathrm{HR} \geq 120$.

Moreover, we calculate the root mean square values (RMS) corresponding to the PR an ST segments. The RMS value of the ST segment is calculated over the signal comprised between the $\mathrm{J}$ point and the ST level measurement point. As regards the RMS value of the PR segment, it was calculated over the signal comprised between the isoelectric measurement point and the fiducial point of the QRS complex.

All the amplitude measures are made with reference to the iso-electric level and using the ECG signal filtered of the baseline noise. The filter applied to the ECG signal is the same used by the ECGPUWAVE tool (a Lynn filter)[4]. The iso-electric and ST level have been averaged over the two adjacent samples in order to minimize the $50 / 60 \mathrm{~Hz}$ noise.

Moreover, the four parameters that the algorithm uses further in order to detect possible ischemic situations are the following ones: RMS of the PR segment, RMS of the ST segment, ST-level and J-level, all of them extracted for each beat detected in the ECG signal, separately.

The four resulting time series are further resampled with a frequency of $2 \mathrm{~Hz}$ and then a smoothing filter is applied to each one. An example of a resampled time series can be seen in figure 1a) meanwhile the figures 1b) - 1e) show an example of the four filtered time series. All the examples are related to the same initial ECG signal.
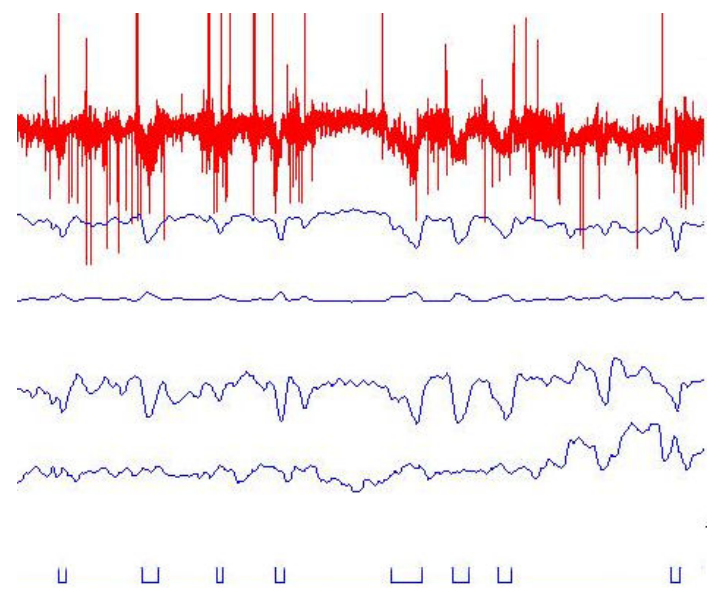

Figure 1. a) resampled ST level time series; b) filtered ST level time series c) filtered RMS(ST) time series d) filtered J level time series; e) filtered RMS(PR) time series; f) correct annotations made by the algorithm 


\subsection{Ischemia detector}

Once the four parameters are extracted, and the corresponding time series are resampled and filtered, the activities shown in the Activity Diagram (figure 2) are applied for each entry set of parameters (two times each second).

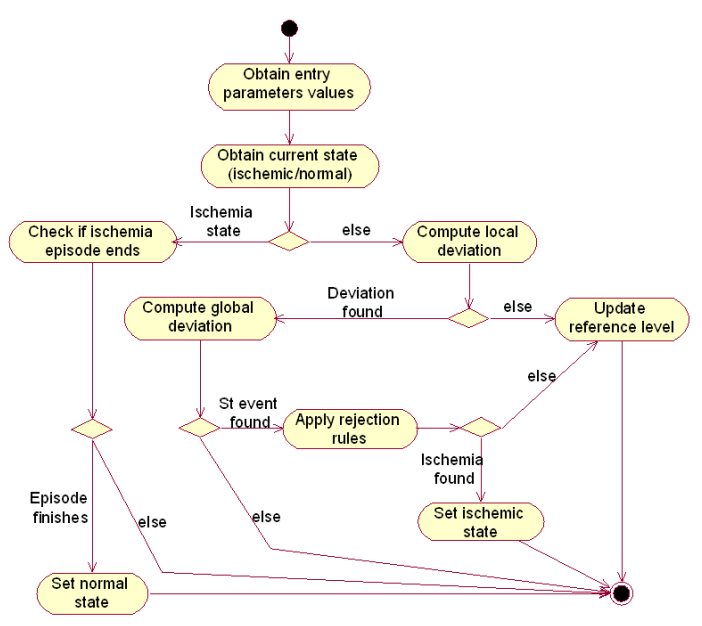

Figure 2. Ischemia Detector's Activity Diagram.

Firstly the algorithm checks the state detected in the previous analysis loop that can be ischemic or normal (Obtain current state, fig 2).

If an ischemic episode was set on, the algorithm checks if the ischemic episode ends (Check if ischemic episode ends, fig 2) and if so, switches the internal state to normal and finishes (Set normal state, fig 2).

Otherwise, if the state was normal, the algorithm decides if the conditions to set on a new ischemic episode are fulfilled. Generally, a new ischemic episode is detected when an important change in the ST level series occurs and none rejection rule is activated. In the following we explain this process with more detail.

First of all, a local ST level deviation is computed (Compute local deviation, fig 2). If this deviation proves to be higher than a fixed threshold, a global ST level deviation with respect to a reference ST level is computed (Compute global deviation, fig 2). The detector contains methods to compute a reference level for the ST level series, reference level that incorporates information about the evolution of the ST level series during larger time intervals (Update reference level, fig 2). If the last deviation fullfills some specified threshold conditions, we say that an ST event has been detected and a set of rejection rules are applied in order to rule out the detected events that are rather due to non ischemic circumstances (Apply rejection rules, fig 2). If no rejection condition has been found for the detected ST event, an ischemic episode is set on (Set ischemic state, fig 2) In the next subsections we explain better some of the methods enumerated before.

\subsubsection{Compute local deviation}

The local deviation is computed over a a fixed window of one minute. If the obtained value does not exceed a threshold $\mathrm{V}_{\text {local }}$ then the algorithm considers that there is no ST event. We chose the value of $\mathrm{V}_{\text {local }}$ to be $5 \mu \mathrm{V}$ during the executions of the algorithm. On the contrary, if the local deviation exceed the mentioned threshold a set of more sophisticated rules are applied as we present below.

\subsubsection{Compute global deviation}

The algorithm calculates the deviation registered from the last tendency change in the ST level time series behaviour (global deviation) and checks if an important ST deviation has occurred. For this to be true the following two rules are to come true:

1. the registered deviation has to diverge from the ST current reference level and

2. a) the deviation relative to the current ST reference has to exceed $50 \mu \mathrm{V}$ or b) the global deviation has to to be higher than $50 \mu \mathrm{V}$ and to coincide with a deviation relative to the ST reference higher than $40 \mu \mathrm{V}$.

The simplicity of these methods ensures that, in normal conditions, when there are no type of oscillation in the ST level, the algorithm performs a minimal computation.

\subsubsection{Update reference level}

As regards the ST reference level estimation, this reference is updated according to the next formula:

$S T_{\text {ref }}[n]=S T_{\text {ref }}[n-1]+\alpha\left(S T_{\text {level }}[n]-S T_{\text {ref }}[n-1]\right)$

where $\alpha$ constant adjusts the speed with which $\mathrm{ST}_{\text {ref }}$ converges to the new $\mathrm{ST}_{\text {level }}$ values. During the execution of the algorithm we set the $\alpha$ to be inverse proportional to the number of samples corresponding to one hour (7200).

Note that the reference is updated only if no local deviation has been found in the ST level series or when an ST event has been found but the rejection rules inferred that the the event is not ischemic. ST reference level computation is freezed when an ischemic episodes is detected or when an ST deviation has been found and the algorithm cannot decide yet if it is ischemic or not. Moreover, small corrections to the $\mathrm{ST}$ reference level are made when an ischemic episode is fired and the ST deviation relative to it is smaller than $50 \mu \mathrm{V}$.

If there is no important ST deviation detected according to the rules enumerated in the 3.3.2 section, the algorithm finishes. On the contrary, if an ST event has been detected, the algorithm will check to see if it is an ischemic ST event or it is rather due to other causes ${ }^{1}$. For this last task we

${ }^{1}$ Note that the algorithm doesn't differentiate the heart-rate related ST changes from ischemic changes. 
have defined a set of rejection rules that we describe further.

\subsubsection{Apply rejection rules}

A first rejection rule is based on comparing the global deviation detected in the ST level series (fig 1b) with the deviation that corresponds to the same time interval in the RMS(ST) series (fig 1c). We observed that important deviations in the ST level time series of ischemic nature can be noticed on a smaller scale in the RMS(ST) series. We rejected to be ischemic that ST level deviations that does not have corresponding deviations in the RMS(ST) time series. In fact, this condition only reinforces the ST events detection, checking to see if the detected ST event is authentic.

The deviation registered in the ST level time series is also compared with that one registered in the $\mathrm{J}$ level time series, rejecting those occurrences whose two deviations were opposite in direction.

The next rejection rule is designed to detect and rule out those ST events that are rather due to body positions changes than ischemic situations. The changes in the body position can affect both ST segment and QRS complex morphology, although the QRS uses to be more sensitive to this kind of changes [6]. For that we compare the global deviation detected in the ST level series (fig 1b) with the deviation that corrresponds to the same time interval in the RMS(PR) series (fig 1e). We reject those episodes with the global deviation in the ST level series lower than $k$ times the deviation registered in the RMS(PR) series for the same time interval. The $k$ parameter is a correction factor which value is about one, with small variations depending on the RMS(PR) value.

Additional checking was made by contrasting the actual ST level deviation with the extreme deviations registered during the last 2 hours, rejecting that episodes that were not following the previous tendency (elevation or depression).

\section{4. $\quad$ Results}

The algorithm described in the previous section was tested over the LTST database recordings [3], the 43 recordings version. An example of the annotations made by the algorithm can be seen in the figure 1f. For the evaluation of the algorithm we used the EVAL_ST tool [7] which allows an objective evaluation of transient ST episode detection algorithms. According to its evaluation criteria, the algorithm's sensitivity is $80.65 \%$ for episodes annotated according to $\mathrm{C}$ protocol and the positive predictivity is $73.11 \%$ for episodes annotated according to A protocol in the LTST database. This means that the algorithm is able to identify the most acute ischemic episodes (defined by the $\mathrm{C}$ protocol), but as regards the predictivity, this re- sult should still be improved. Nevertheless we want to notice that the algorithm can be run in a PDA in real time. The optimal processing cicle duration proved is of $2 \mathrm{sec}$ onds for one channel analysis which implies an ischemia detection delay between 4 and 5 seconds.

\section{Discussion and conclusions}

An algorithm designed to detect transient ischemic episodes in real time has been developed and tested against part of the LTST database. The algorithm is able to detect the most critical ischemic episodes. Moreover, as far as we know there are still few algorithms for real time detection of ischemic episodes and no one for the LTST database. Furthermore, the algorithm provides efficient performance during its execution in real time on a PDA. The use of the entire database could help to improve these results.

\section{References}

[1] Smrdel A, Jager F. Automated detection of transient STsegment episodes in 24h electrocardiograms. Med Biol Eng Comput 2004;42:303-311.

[2] Stadler R, Lu S, Nelson S, Stylos L. A real-time ST-segment monitoring algorithm for implantable devices. J Electrocardiology 2001;34:119-126.

[3] Jager F, Taddei A, Moody GB, Emdin M, Antolic, Dorn R, Smrdel A, Marchesi C, Mark R. Long-term ST database: a reference for the development and evaluation of automated ischaemia detectors and for study of the dynamics of myocardial ischaemia. Med Biol Eng Comput 2003;41:172-182.

[4] Jané R, Blasi A, García J, Laguna P. Evaluation of an automatic threshold based detector of waveform limits in holter ecg with the qt database. Computers in Cardiology 1997; 24:295-298.

[5] Rodríguez J, Goñi A, Illarramendi A. Real-time classification of ECG on a PDA. IEEE Transactions of Informations Technology in Biomedicine 2005;9:23-34.

[6] Adams MG, Drew BJ. Body position effects on the ECG: Implication for ischemia monitoring. J Electrocardiology 1997; 30:285-291.

[7] Jager F, Smrdel A, Mark RG. An open-source tool to evaluate performance of transient ST segment episode detection algorithms. In Computers in Cardiology 2004. IEEE Press, 2004; 585-588.

Address for correspondence:

Alfredo Goñi

University of The Basque Country

Spain, Donostia - San Sebastián

http://siul02.si.ehu.es/

tel./fax.: ++34-943-01-5066/5590 